\title{
RAINBOW DECOMPOSITIONS
}

\author{
RAPHAEL YUSTER
}

(Communicated by Jim Haglund)

\begin{abstract}
A rainbow coloring of a graph is a coloring of the edges with distinct colors. We prove the following extension of Wilson's Theorem. For every integer $k$ there exists an $n_{0}=n_{0}(k)$ so that for all $n>n_{0}$, if$$
n \bmod k(k-1) \in\{1, k\},
$$

then every properly edge-colored $K_{n}$ contains $\left(\begin{array}{c}n \\ 2\end{array}\right) /\left(\begin{array}{c}k \\ 2\end{array}\right)$ pairwise edge-disjoint rainbow copies of $K_{k}$.

Our proof uses, as a main ingredient, a double application of the probabilistic method.
\end{abstract}

\section{INTRODUCTION}

All graphs considered here are finite, undirected, and simple. For standard graph-theoretic terminology the reader is referred to [2]. For an integer $k \geq 3$, a Steiner system $S(2, k, n)$ is a set $X$ of $n$ points, and a collection of subsets of $X$ of size $k$ (called blocks), such that any two points of $X$ are in exactly one of the blocks. We say that the complete graph $K_{n}$ has a $K_{k}$-decomposition if $K_{n}$ contains $\left(\begin{array}{l}n \\ 2\end{array}\right) /\left(\begin{array}{l}k \\ 2\end{array}\right)$ pairwise edge-disjoint copies of $K_{k}$. Clearly, an $S(2, k, n)$ exists if and only if $K_{n}$ has a $K_{k}$-decomposition. More generally, for a given graph $H$ we say that $K_{n}$ is $H$-decomposable if $K_{n}$ contains $\left(\begin{array}{c}n \\ 2\end{array}\right) / e(H)$ edge-disjoint copies of $H$.

For a graph $H$, let $\operatorname{gcd}(H)$ denote the largest integer that divides the degree of each vertex of $H$. Two obvious necessary conditions for the existence of an $H$ decomposition of $K_{n}$ are that $e(H)$ divides $\left(\begin{array}{l}n \\ 2\end{array}\right)$ and that $\operatorname{gcd}(H)$ divides $n-1$ (notice that $\left.n-1=\operatorname{gcd}\left(K_{n}\right)\right)$. These trivial divisibility conditions are not always sufficient. For example $K_{4}$ does not have a $K_{1,3}$-decomposition. However, a seminal result of Wilson [10] show that, for all $n$ sufficiently large, the two divisibility conditions suffice.

Theorem 1.1 (Wilson [10]). For every fixed graph $H$ there exists $n_{0}=n_{0}(H)$ so that if $n>n_{0}, e(H) \mid\left(\begin{array}{c}n \\ 2\end{array}\right)$, and $\operatorname{gcd}(H) \mid n-1$, then $K_{n}$ has an $H$-decomposition.

We note that if $H=K_{k}$ the divisibility conditions translate to $n \bmod k(k-1) \in$ $\{1, k\}$. The case $k=3$ (i.e. the existence of a Steiner triple system) is an old result. Such a system exists if and only if $n \bmod 6 \in\{1,3\}$ [9]. The case $k=4$ is also known to exist if and only if $n \bmod 12 \in\{1,4\}[3]$.

Recently there has been a rise of interest in extremal rainbow-type problems. A rainbow coloring of a graph is a coloring of the edges with distinct colors. An edge

Received by the editors September 28, 2006.

2000 Mathematics Subject Classification. Primary 05C15, 05C70, 05B40, 03 E05. 
coloring of a graph is called proper if two edges sharing an endpoint receive distinct colors. Vizing's theorem asserts that there exists a proper edge coloring of a graph $G$ which uses at most $\Delta(G)+1$ colors. In extremal graph theory, one is interested in establishing conditions on a graph $G$ that guarantee the existence of a (possibly induced) set of subgraphs of a specific type (Ramsey and Turán type problems are central problems of this type). In rainbow-type problems one is interested in establishing conditions on a properly edge-colored graph $G$ that guarantee the existence of a (possibly induced) set of rainbow subgraphs of a specific type. Many graph theoretic parameters have corresponding rainbow variants. Erdös and Rado [6] were among the first to consider problems of this type. Jamison, Jiang and Ling [7], and Chen, Schelp and Wei [4] considered Ramsey type variants where an arbitrary number of colors can be used. Alon et al. [1] studied the function $f(H)$ which is the minimum integer $n$ such that any proper edge coloring of $K_{n}$ has a rainbow copy of $H$. Keevash et al. [8] considered the rainbow Turán number $e x^{*}(n, H)$ which is the largest integer $m$ such that there exists a properly edgecolored graph with $n$ vertices and $m$ edges and which has no rainbow copy of $H$. Yuster [12] gave necessary and sufficient conditions for the existence of rainbow $H$-factors.

Is Theorem 1.1 still true in the rainbow setting? The main result of this paper shows that, indeed, this is the case.

Theorem 1.2. For every fixed graph $H$ there exists $n_{1}=n_{1}(H)$ so that if $n>$ $n_{1}, e(H) \mid\left(\begin{array}{l}n \\ 2\end{array}\right)$, and $\operatorname{gcd}(H) \mid n-1$, then a properly edge-colored $K_{n}$ has an $H$ decomposition so that each copy of $H$ in the decomposition is rainbow colored.

We note that the case $H=K_{3}$ is trivial since every properly edge-colored triangle is also rainbow colored. However, already for $K_{4}$, the analogue of Brouwer's result [3] trivially does not hold, as a properly edge-colored $K_{4}$ need not be rainbow colored. The proof of Theorem 1.2 appears in Section 3 and is based upon a double application of the probabilistic method. A few lemmas that are needed for the proof of Theorem 1.2 follow in the next section. The final section contains some concluding remarks and open problems.

\section{Preliminary LEMmas}

Let $F$ be a family of positive integers. We say that $K_{n}$ is $F$-decomposable if we can color the edges of $K_{n}$ such that each color class induces a complete graph whose order belongs to $F$.

Let $H$ be a fixed graph, and let $t$ be a positive integer. We say that a finite set of positive integers $F$ is an $(H, t)$-complete decomposition set $((H, t)$-CDS for short) if the following holds:

1. If $k \in F$, then $k \geq t$ and $K_{k}$ is $H$-decomposable.

2. There exists $N$ such that for all $n>N, K_{n}$ is $H$-decomposable if and only if $K_{n}$ is $F$-decomposable.

We prove that, for all $H$ and $t$, an $(H, t)$-CDS always exists. For this purpose we need a theorem of Wilson on $F$-decompositions. For a (possibly infinite) set of positive integers $F$, let $\operatorname{gcd}(F)$ denote the largest positive integer which divides each number in $F$, let $F_{1}=\{n-1 \mid n \in F\}$ and let $F_{2}=\{n(n-1) / 2 \mid n \in F\}$. In [11] Wilson has proved the following: 
Lemma 2.1 (Wilson [11]). Let $F$ be a finite set of positive integers. Then, there exists $n_{0}=n_{0}(F)$ such that if $n>n_{0}, \operatorname{gcd}\left(F_{1}\right)$ divides $n-1$ and $\operatorname{gcd}\left(F_{2}\right)$ divides $\left(\begin{array}{l}n \\ 2\end{array}\right)$, then there exists an $F$-decomposition of $K_{n}$.

We can now show the following:

Lemma 2.2. Let $H$ be a graph, and let $t$ be a positive integer. Then, an $(H, t)-C D S$ exists.

Proof. Let

$$
S=\left\{s \mid s \geq t \text { and } K_{s} \text { is } H \text {-decomposable }\right\} .
$$

$S$ is infinite but, obviously, $\operatorname{gcd}\left(S_{1}\right)$ and $\operatorname{gcd}\left(S_{2}\right)$ are finite. Thus, there are finite subsets $S^{\alpha} \subset S$ and $S^{\beta} \subset S$ such that $\operatorname{gcd}\left(S_{1}^{\alpha}\right)=\operatorname{gcd}\left(S_{1}\right)$ and $\operatorname{gcd}\left(S_{2}^{\beta}\right)=\operatorname{gcd}\left(S_{2}\right)$. Let $F=S^{\alpha} \cup S^{\beta}$. Note that since $S^{\alpha} \subset F$ then $\operatorname{gcd}\left(F_{1}\right)$ divides $\operatorname{gcd}\left(S_{1}\right)$. Similarly, since $S^{\beta} \subset F$ we have that $\operatorname{gcd}\left(F_{2}\right)$ divides $\operatorname{gcd}\left(S_{2}\right)$. We claim that $F$ is an $(H, t)$ CDS. First note that, by definition, every $s \in F$ satisfies $s \geq t$ and $K_{s}$ is $H$ decomposable. Now let $N=\max \left\{n_{0}, t\right\}$ where $n_{0}=n_{0}(F)$ is the constant defined in the statement of Lemma 2.1. It suffices to show that for every $n>N$, if $K_{n}$ is $H$-decomposable, then it is also $F$-decomposable. Indeed, if $K_{n}$ is $H$-decomposable, then $n \in S$, so $\operatorname{gcd}\left(F_{1}\right)\left|\operatorname{gcd}\left(S_{1}\right)\right| n-1$, and $\operatorname{gcd}\left(F_{2}\right)\left|\operatorname{gcd}\left(S_{2}\right)\right|\left(\begin{array}{l}n \\ 2\end{array}\right)$. Thus, by Lemma $2.1, K_{n}$ is $F$-decomposable.

A properly colored forest $T$ will be called a weed if it contains three distinct edges $e_{1}, e_{2}, e_{3}$, so that for each $e_{i}$ there is an edge $f_{i} \notin\left\{e_{1}, e_{2}, e_{3}\right\}$ having the same color as $e_{i}$ (the $f_{i}$ need not be distinct; in particular, if $e_{1}, e_{2}, e_{3}$ have the same color we can have $f_{1}=f_{2}=f_{3}$ ). Furthermore, we assume that $T$ has no sub-weed. Trivially, every weed contains at most 6 edges and at least 4 edges. In fact, is is not difficult to check that, up to color isomorphism, there is precisely one weed with four edges, 8 weeds with five edges, and 41 weeds with six edges. The 50 weeds are shown in Figure 1.

A properly colored graph is called multiply colored if no color appears only once in the graph. We need the following combinatorial lemma.

Lemma 2.3. Every multiply colored graph with at least 29 edges contains a weed.

Proof. Let $G$ be a multiply colored graph with 29 edges. If some color appears four times in $G$, then it forms a matching with four edges which is the weed $W_{1}$. Otherwise, suppose that some color $c$ appears three times in the edges $\left(v_{1}, v_{2}\right),\left(v_{3}, v_{4}\right)$, $\left(v_{5}, v_{6}\right)$. Since six vertices induce at most 15 edges, there is some edge $(x, y)$ colored with $c^{\prime}$, and $x \notin\left\{v_{1}, v_{2}, v_{3}, v_{4}, v_{5}, v_{6}\right\}$. Let $(w, z)$ be another edge colored with $c^{\prime}$. Since the coloring of $G$ is proper, the five edges $\left(v_{1}, v_{2}\right),\left(v_{3}, v_{4}\right),\left(v_{5}, v_{6}\right),(x, y),(w, z)$ form a weed.

We remain with the case where each color appears precisely twice in $G$. Let $c$ be the color of $\left(v_{1}, v_{2}\right)$ and of $\left(v_{3}, v_{4}\right)$. Let $c^{\prime}$ be a color not appearing in the subgraph induced by $\left\{v_{1}, v_{2}, v_{3}, v_{4}\right\}$. Denote the edges colored with $c^{\prime}$ by $\left(v_{5}, v_{6}\right)$ and $\left(v_{7}, v_{8}\right)$. Assume first that $\left\{v_{1}, v_{2}, v_{3}, v_{4}\right\} \cap\left\{v_{5}, v_{6}, v_{7}, v_{8}\right\}=\emptyset$. Since 8 vertices induce at most 28 edges, there is some edge $(x, y)$ colored with $c^{\prime \prime}$, and $x \notin$ $\left\{v_{1}, v_{2}, v_{3}, v_{4}, v_{5}, v_{6}, v_{7}, v_{8}\right\}$. Let $(w, z)$ be another edge colored with $c^{\prime \prime}$. Since the coloring of $G$ is proper, the six edges $\left(v_{1}, v_{2}\right),\left(v_{3}, v_{4}\right),\left(v_{5}, v_{6}\right),\left(v_{7}, v_{8}\right),(x, y),(w, z)$ form a weed.

Assume next that $\left|\left\{v_{1}, v_{2}, v_{3}, v_{4}\right\} \cap\left\{v_{5}, v_{6}, v_{7}, v_{8}\right\}\right|=1$. Without loss of generality, $v_{1}=v_{5}$. Since 7 vertices induce at most 21 edges, there is some edge $(x, y)$ 


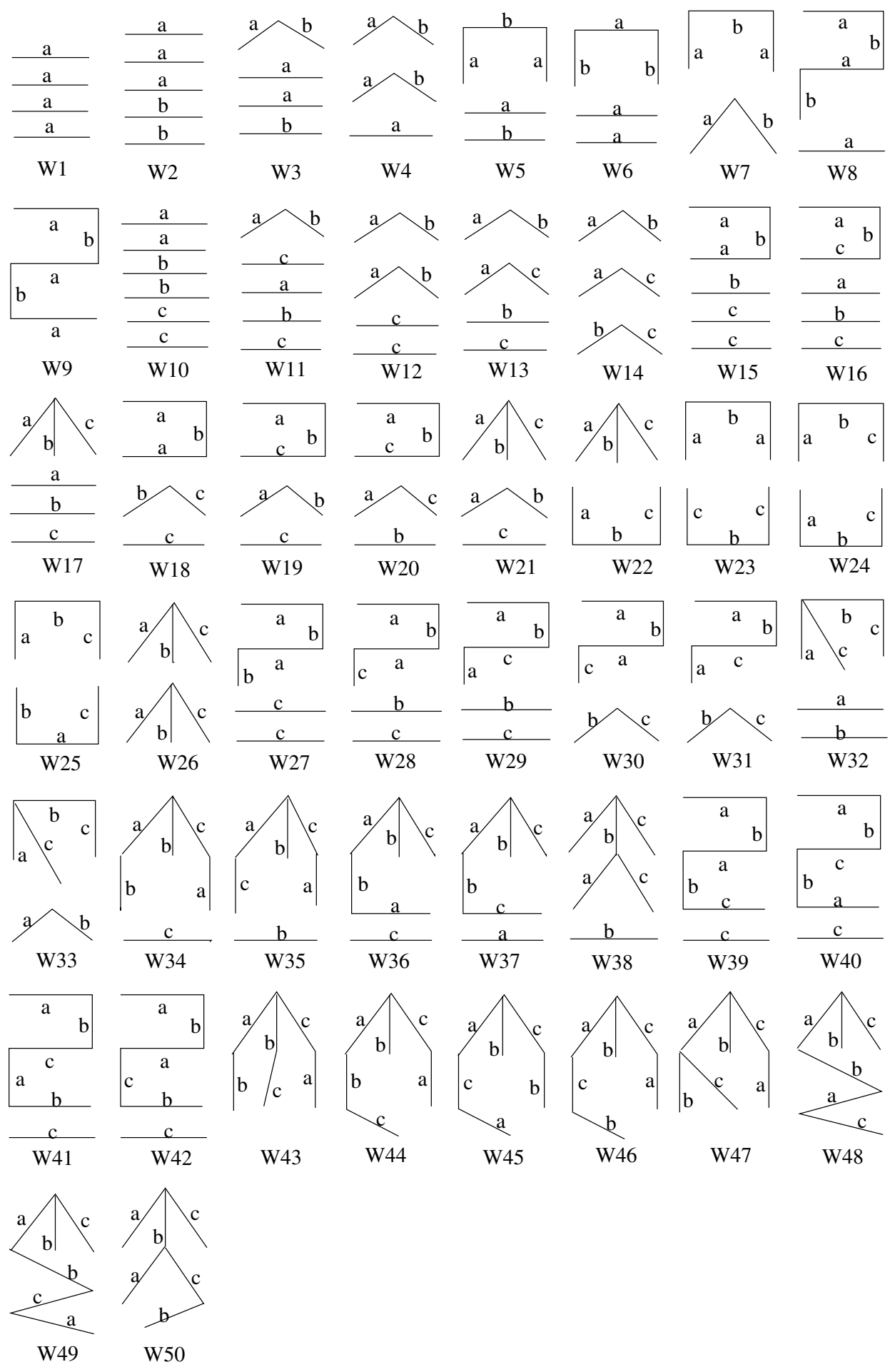

Figure 1. The 50 weeds. Labels on edges represent colors 
colored with $c^{\prime \prime}$ so that $c^{\prime \prime}$ is not the color of the edge $\left(v_{2}, v_{6}\right)$ (if the latter even exists), and $x \notin\left\{v_{1}, v_{2}, v_{3}, v_{4}, v_{6}, v_{7}, v_{8}\right\}$. Let $(w, z)$ be another edge colored with $c^{\prime \prime}$. The six edges $\left(v_{1}, v_{2}\right),\left(v_{3}, v_{4}\right),\left(v_{1}, v_{6}\right),\left(v_{7}, v_{8}\right),(x, y)$, and $(w, z)$, form a weed.

We remain with the case $\left|\left\{v_{1}, v_{2}, v_{3}, v_{4}\right\} \cap\left\{v_{5}, v_{6}, v_{7}, v_{8}\right\}\right|=2$. There are two subcases here, up to isomorphism. The first is $v_{1}=v_{5}$ and $v_{3}=v_{7}$. The second is $v_{1}=$ $v_{5}$ and $v_{2}=v_{7}$. In the first sub-case, since six vertices induce at most 15 edges, there is some edge $(x, y)$ colored with $c^{\prime \prime}$ so that $c^{\prime \prime}$ is not the color of the edge $\left(v_{2}, v_{6}\right)$ nor of the edge $\left(v_{4}, v_{8}\right)$ (if any of these edges even exist), and $x \notin\left\{v_{1}, v_{2}, v_{3}, v_{4}, v_{6}, v_{8}\right\}$. Let $(w, z)$ be another edge colored with $c^{\prime \prime}$. Since the coloring of $G$ is proper, the six edges $\left(v_{1}, v_{2}\right),\left(v_{3}, v_{4}\right),\left(v_{1}, v_{6}\right),\left(v_{3}, v_{8}\right),(x, y),(w, z)$ form a weed. Similarly, in the second sub-case, there is some edge $(x, y)$ colored with $c^{\prime \prime}$ so that $c^{\prime \prime}$ is not the color of the edge $\left(v_{2}, v_{6}\right)$ nor of the edge $\left(v_{1}, v_{8}\right)$ nor of the edge $\left(v_{6}, v_{8}\right)$ (if any of these edges even exist), and $x \notin\left\{v_{1}, v_{2}, v_{3}, v_{4}, v_{6}, v_{8}\right\}$. Let $(w, z)$ be another edge colored with $c^{\prime \prime}$. The six edges $\left(v_{1}, v_{2}\right),\left(v_{3}, v_{4}\right),\left(v_{1}, v_{6}\right),\left(v_{2}, v_{8}\right),(x, y),(w, z)$ form a weed.

It is possible to improve the constant 29 in Lemma 2.3 at the price of a tedious case analysis. This, however, is not important for our purposes. A multiple coloring of $K_{6}$ (which has 15 edges) having no weed is easily obtained by coloring a matching of three edges red, and the other 12 edges with 6 distinct colors, where the union of each of these colors with the red edges contains a $C_{4}$.

\section{Proof of the MAIN RESUlt}

The proof of Theorem 1.2 is established by the following two lemmas.

Lemma 3.1. For a positive integer $r$, and for a graph $H$, there is a constant $C=C(r, H)$ so that if $k>C$ and $K_{k}$ is $H$-decomposable, then for any given set $U$ of $r$ edges of $K_{k}$, there is an $H$-decomposition of $K_{k}$ in which no two elements of $U$ appear together in the same $H$-copy of the decomposition.

Proof. Suppose $K_{k}$ is $H$-decomposable, and fix an $H$-decomposition of $K_{k}$, denoted $L$. Let $\pi$ be a permutation of $\{1, \ldots, k\}$. The permutation $\pi$ and $L$ naturally define another $H$-decomposition of $K_{k}$, denoted $L_{\pi}$. Indeed, if $Q \in L$ is a copy of $H$, then $Q$ is mapped to a copy $Q_{\pi} \in L_{\pi}$ by the automorphism $\pi$ on the vertex set of $K_{k}$.

Fix a set $U$ of $r$ edges of $K_{k}$. Picking $\pi$ uniformly at random, consider the probability that two edges $e, f \in U$ are in the same copy of $H$ in $L_{\pi}$. If $e$ and $f$ do not share an endpoint, the probability of this event is at most

$$
\frac{e(H)-1}{\left(\begin{array}{c}
k-2 \\
2
\end{array}\right)} \text {. }
$$

If $e$ and $f$ share an endpoint, the probability of this event is at most

$$
\frac{v(H)-2}{k-2} \text {. }
$$

As there are $\left(\begin{array}{l}n \\ 2\end{array}\right)$ possible pairs of edges of $U$, we have that, as long as

$$
\left(\begin{array}{l}
r \\
2
\end{array}\right) \max \left\{\frac{e(H)-1}{\left(\begin{array}{c}
k-2 \\
2
\end{array}\right)}, \frac{v(H)-2}{k-2}\right\}<1,
$$


with positive probability, no two elements of $U$ appear together in the same $H$-copy of $L_{\pi}$. Thus, for sufficiently large $k$, as a function of $r$ and $H$, inequality (1) holds, and hence the lemma follows.

Lemma 3.2. For every finite set of positive integers $F$ there exists a constant $M=M(F)$, so that for every $n>M$, if $K_{n}$ is a properly edge colored and $F$ decomposable, then $K_{n}$ also has an $F$-decomposition so that every element of the decomposition contains no weed.

Proof. Suppose that $K_{n}$ is properly edge colored and $F$-decomposable, and fix an $F$-decomposition of $K_{n}$, denoted $L$. Notice that, clearly, $|L| \leq\left(\begin{array}{l}n \\ 2\end{array}\right)$. As in the proof of Lemma 3.1, let $\pi$ be a permutation of $\{1, \ldots, n\}$, and let $L_{\pi}$ be the $F$ decomposition naturally defined by $\pi$ and $L$. It suffices to prove that if $\pi$ is picked uniformly at random, then for each $Q \in L$, the mapped copy $Q_{\pi} \in L_{\pi}$ contains a weed with probability less than $1 / n^{2}$ (since $1 / n^{2}<1 /|L|$ ), assuming $n$ is sufficiently large as a function of $F$. Let $z$ be the largest integer in $F$.

Fix $Q \in L$, and suppose that $|Q|=q \leq z$. Without loss of generality, $Q$ is a $K_{q}$ induced by the vertices $\{1, \ldots, q\}$. We will prove that, for each possible weed $W_{i}$ for $i=1, \ldots, 50$, the probability that $Q_{\pi}$ (which is a properly colored $K_{q}$ subgraph of $\left.K_{n}\right)$ contains a forest which is color isomorphic to $W_{i}$ is less than $1 /\left(50 n^{2}\right)$. For the sake of space compactness, we will prove this for $W_{1}$ (the only weed with four edges), for $W_{7}$ (a representative weed with five edges), and for $W_{33}$ (a representative weed with six edges). The proof for all other 47 weeds is practically the same.

Consider a labeled copy of a forest that is isomorphic to the uncolored forest $W_{1}$ in $Q$. As $W_{1}$ contains 8 vertices, there are less than $q^{8}$ such labeled forests. Fixing one such forest $P$, w.l.o.g. the one defined by the edges $(1,2),(3,4),(5,6),(7,8)$, we will prove that all the edges of $P_{\pi}$ are colored the same with probability less than $1 /\left(50 n^{2} q^{8}\right)$. This will show that the probability that $Q_{\pi}$ contains the weed $W_{1}$ is less than $1 /\left(50 n^{2}\right)$, as required. Let $a$ be the color of $(\pi(1), \pi(2))$. Given $a$, the probability that $(\pi(3), \pi(4))$ is also colored $a$ is precisely

$$
\frac{n_{a}-1}{\left(\begin{array}{c}
n-2 \\
2
\end{array}\right)} \leq \frac{n / 2-1}{\left(\begin{array}{c}
n-2 \\
2
\end{array}\right)}=\frac{1}{n-3}
$$

where $n_{a}$ is the number of edges colored $a$ in the given proper edge coloring of $K_{n}$. Similarly, given that $(\pi(1), \pi(2))$ and $(\pi(3), \pi(4))$ are both colored $a$, the probability that $(\pi(5), \pi(6))$ is also colored $a$ is precisely $\left(n_{a}-2\right) /\left(\begin{array}{c}n-4 \\ 2\end{array}\right) \leq 1 /(n-5)$. Finally, given that $(\pi(1), \pi(2)),(\pi(3), \pi(4))$, and $(\pi(5), \pi(6))$ are all colored $a$, the probability that $(\pi(7), \pi(8))$ is also colored $a$ is precisely $\left(n_{a}-3\right) /\left(\begin{array}{c}n-6 \\ 2\end{array}\right) \leq 1 /(n-7)$. Overall, the edges of $P_{\pi}$ are colored the same with probability at most

$$
\frac{1}{(n-3)(n-5)(n-7)} \leq \frac{1}{50 n^{2} z^{8}} \leq \frac{1}{50 n^{2} q^{8}}
$$

if $n$ is sufficiently large, as required.

Consider a labeled copy of a forest which is isomorphic to the uncolored forest $W_{7}$ in $Q$. As $W_{7}$ contains 7 vertices, there are less than $q^{7}$ such labeled forests. Fixing one such forest $P$, w.l.o.g. the one defined by the edges $(1,2),(2,3),(3,4),(5,6)$, $(6,7)$ (notice that this is indeed a labeled copy of $W_{7}$ ), we will prove that the probability that all the edges $(\pi(1), \pi(2)),(\pi(3), \pi(4)),(\pi(5), \pi(6))$ are colored the same, and that edges $(\pi(2), \pi(3))$ and $(\pi(6), \pi(7))$ are also colored the same is less than $1 /\left(50 n^{2} q^{7}\right)$. This will show that the probability that $Q_{\pi}$ contains the weed 
$W_{7}$ is less than $1 /\left(50 n^{2}\right)$, as required. Let $a$ be the color of $(\pi(1), \pi(2))$ and let $b$ be the color of $(\pi(2), \pi(3))$. Notice that $a \neq b$ since the given coloring of $K_{n}$ is proper. Given $a$ and $b$, the probability that $(\pi(3), \pi(4))$ is colored $a$ is at most $1 /(n-3)$. This is because at most one edge $(\pi(3), x)$ incident with $\pi(3)$ is colored $a$, and the probability that $x=\pi(4)$ is precisely $1 /(n-3)$. Similarly, given that $(\pi(1), \pi(2))$ and $(\pi(3), \pi(4))$ are both colored $a$, and that $(\pi(2), \pi(3))$ is colored $b$, the probability that $(\pi(5), \pi(6))$ is colored $a$ is precisely $\left(n_{a}-2\right) /\left(\begin{array}{c}n-4 \\ 2\end{array}\right) \leq 1 /(n-5)$. Finally, given that $(\pi(1), \pi(2)),(\pi(3), \pi(4))$, and $(\pi(5), \pi(6))$ are colored $a$, and that $(\pi(2), \pi(3))$ is colored $b$, the probability that $(\pi(6), \pi(7))$ is colored $b$ is at most $1 /(n-6)$. This is because at most one edge $(\pi(6), x)$, where $x \neq \pi(i)$ for $i=1, \ldots, 5$, incident with $\pi(6)$, is colored $b$, and the probability that $x=\pi(7)$ is precisely $1 /(n-6)$. Overall, the probability that $(\pi(1), \pi(2)),(\pi(3), \pi(4)),(\pi(5), \pi(6))$ are colored the same, and that $(\pi(2), \pi(3))$ and $(\pi(6), \pi(7))$ are also colored the same is at most

$$
\frac{1}{(n-3)(n-5)(n-6)} \leq \frac{1}{50 n^{2} z^{7}} \leq \frac{1}{50 n^{2} q^{7}}
$$

if $n$ is sufficiently large, as required.

Consider a labeled copy of a forest which is isomorphic to the uncolored forest $W_{33}$ in $Q$. As $W_{33}$ contains 8 vertices, there are less than $q^{8}$ such labeled forests. Fixing one such forest $P$, w.l.o.g. the one defined by the edges $(1,2),(2,3),(3,4)$, $(2,5),(6,7),(7,8)$ (notice that this is indeed a labeled copy of $\left.W_{33}\right)$, we will prove that the probability that the edges $(\pi(1), \pi(2))$ and $(\pi(6), \pi(7))$ are colored the same, the edges $(\pi(2), \pi(3))$ and $(\pi(7), \pi(8))$ are colored the same, and the edges $(\pi(3), \pi(4))$ and $(\pi(2), \pi(5))$ are colored the same, is less than $1 /\left(50 n^{2} q^{8}\right)$. This will show that the probability that $Q_{\pi}$ contains the weed $W_{33}$ is less than $1 /\left(50 n^{2}\right)$, as required. Let $a$ be the color of $(\pi(1), \pi(2))$, let $b$ be the color of $(\pi(2), \pi(3))$, and let $c$ be the color of $(\pi(2), \pi(5))$. Notice that $a, b$, and $c$ are distinct colors since the given coloring of $K_{n}$ is proper. Given $a, b$, and $c$, the probability that $(\pi(3), \pi(4))$ is colored $c$ is at most $1 /(n-4)$. Similarly, given that $(\pi(2), \pi(5))$ and $(\pi(3), \pi(4))$ are both colored $c$, that $(\pi(1), \pi(2))$ is colored $a$, and that $(\pi(2), \pi(3))$ is colored $b$, the probability that $(\pi(6), \pi(7))$ is colored $a$ is at most $((n-5) / 2) /\left(\begin{array}{c}n-5 \\ 2\end{array}\right) \leq 1 /(n-6)$. Finally, given that $(\pi(2), \pi(5))$ and $(\pi(3), \pi(4))$ are both colored $c$, that $(\pi(1), \pi(2))$ and $(\pi(6), \pi(7))$ are both colored $a$, and that $(\pi(2), \pi(3))$ is colored $b$, the probability that $(\pi(7), \pi(8))$ is colored $b$ is at most $1 /(n-7)$. Overall, the probability that the edges $(\pi(1), \pi(2))$ and $(\pi(6), \pi(7))$ are colored the same, the edges $(\pi(2), \pi(3))$ and $(\pi(7), \pi(8))$ are colored the same, and the edges $(\pi(3), \pi(4))$ and $(\pi(2), \pi(5))$ are colored the same is at most

$$
\frac{1}{(n-4)(n-6)(n-7)} \leq \frac{1}{50 n^{2} z^{8}} \leq \frac{1}{50 n^{2} q^{8}}
$$

if $n$ is sufficiently large, as required.

Proof of Theorem 1.2. Fix a graph $H$, and let $t=C(28, H)$ be the constant from Lemma 3.1. Let $F$ be an $(H, t)$-CDS, whose existence is guaranteed by Lemma 2.2. Let $M=M(F)$ be the constant from Lemma 3.2. Since $F$ is an $(H, t)$-CDS, there exists $N=N(F)$ so that for all $n>N, K_{n}$ is $H$-decomposable if and only if $K_{n}$ is $F$-decomposable. We define

$$
n_{1}=n_{1}(H)=\max \left\{M, N, n_{0}(H)\right\}
$$

where $n_{0}(H)$ is the constant from Theorem 1.1. 
Suppose that $n>n_{1}, e(H) \mid\left(\begin{array}{l}n \\ 2\end{array}\right)$, and $\operatorname{gcd}(H) \mid n-1$ and consider a properly edgecolored $K_{n}$. Since $n_{1} \geq n_{0}$ we have, by Theorem 1.1 , that $K_{n}$ is $H$-decomposable. Since $n_{1} \geq N$ we have, by the definition of $F$, that $K_{n}$ is also $F$-decomposable. Since $n_{1} \geq M$ we have, by Lemma 3.2, that there is an $F$-decomposition of $K_{n}$ so that every element of the decomposition contains no weed. Consider some $K_{k}$ element of such an $F$-decomposition. Thus, $k \in F$ and hence $k \geq t$. Furthermore, $K_{k}$ is $H$-decomposable. Let $U$ be a maximal multiply colored subgraph of $K_{k}$. We identify $U$ with its set of edges. Since $K_{k}$ contains no weed, we have, by Lemma 2.3 , that $|U| \leq 28$. Since $k \geq t=C(28, H)$ we have, by Lemma 3.1, that $K_{k}$ has an $H$-decomposition so that no two edges of $U$ appear together in the same $H$-copy of the decomposition. But this implies that each copy of $H$ in such a decomposition is rainbow colored. Repeating this process for each element of the $F$-decomposition yields an $H$-decomposition of $K_{n}$ in which each element is rainbow colored.

\section{Concluding Remarks}

- The statement of Theorem 1.2 remains valid even if $K_{n}$ is not necessarily properly colored, but, instead, no color appears more than constantly many times in the edges incident with a vertex. More precisely, an edge coloring is $C$-proper if the subgraph induced by each color has maximum degree at most $C$. The proof is essentially the same, although one has to broaden the definition of weeds to forests that are not necessarily properly colored, but $C$-properly colored (there are still finitely many such weeds). The following extension of Theorem 1.2 is:

Theorem 4.1. For every fixed graph $H$ and positive integer $C$ there exists $n_{1}=n_{1}(H, C)$ so that if $n>n_{1}, e(H) \mid\left(\begin{array}{l}n \\ 2\end{array}\right)$, and $\operatorname{gcd}(H) \mid n-1$, then a $C$-properly edge-colored $K_{n}$ has an $H$-decomposition so that each copy of $H$ in the decomposition is rainbow colored.

- The exact smallest possible value of $n_{1}=n_{1}(H)$ is extremely difficult to determine, even for the smallest non-trivial cases. For example, is it true that every properly edge colored $K_{13}$ contains 13 rainbow copies of $K_{4}$ ? It is not difficult to show that this is true if each color is used at most twice, but already in $K_{13}$ we can have each color appearing 6 times. We leave this as an open problem.

\section{REFERENCES}

[1] N. Alon, T. Jiang, Z. Miller and D. Pritikin, Properly colored subgraphs and rainbow subgraphs in edge-colorings with local constraints, Random Struct. Algorithms 23 (2003), No. 4, 409-433. MR2016871 (2004i:05106)

[2] B. Bollobás, Modern Graph Theory, Springer-Verlag, 1998. MR1633290 (99h:05001)

[3] A.E. Brouwer, Optimal packing of $K_{4}$ 's into a $K_{n}$, J. Combin. Theory, Ser. A 26 (1979), 278-297. MR535158 (80j:05049)

[4] G. Chen, R. Schelp and B. Wei, Monochromatic - rainbow Ramsey numbers, 14th Cumberland Conference Abstracts. Posted at "http://www.msci.memphis.edu/ balistep/Abstracts.html".

[5] C.J. Colbourn and J.H. Dinitz, CRC Handbook of Combinatorial Design, CRC Press 1996. MR1392993 (97a:05001)

[6] P. Erdős and R. Rado, A combinatorial theorem, J. London Math. Soc. 25 (1950), 249-255. MR0037886 (12:322f)

[7] R. Jamison, T. Jiang and A. Ling, Constrained Ramsey numbers, J. Graph Theory 42 (2003), No. 1, 1-16. MR1943103 (2003m:05129) 
[8] P. Keevash, D. Mubayi, B. Sudakov and J. Verstraete, Rainbow Turán Problems, Combinatorics, Probability, and Computing 16 (2007), 109-126. MR2286514 (2007i:05092)

[9] T.P. Kirkman, On a Problem in Combinatorics, Cambridge Dublin Math. J. 2 (1847), 191204.

[10] R. M. Wilson, Decomposition of complete graphs into subgraphs isomorphic to a given graph, Congressus Numerantium XV (1975), 647-659. MR0396347 (53:214)

[11] R. M. Wilson, An existence theory for pairwise balanced designs II. The structure of PBDclosed sets and the existence conjectures, J. Combin. Theory, Ser. A 13 (1972), 246-273. MR0304204 (46:3339)

[12] R. Yuster, Rainbow H-factors, The Electronic Journal of Combinatorics 13 (2006), \#R13. MR2200541 (2006k:05111)

Department of Mathematics, University of Haifa, Haifa 31905, Israel

E-mail address: raphy@math.haifa.ac.il 